CLINICAL STUDY

\title{
Real-time PCR provides evidence for thyrotropin receptor mRNA expression in orbital as well as in extraorbital tissues
}

Patrizia Agretti, Luca Chiovato ${ }^{1}$, Giuseppina De Marco, Claudio Marcocci, Barbara Mazzi, Stefano Sellari-Franceschini, Paolo Vitti, Aldo Pinchera and Massimo Tonacchera

Dipartimento di Endocrinologia e Metabolismo, Ortopedia e Traumatologia, Medicina del Lavoro, Università di Pisa, Pisa, Italy and ${ }^{1}$ Cattedra di Endocrinologia, Fondazione S Maugeri IRCCS, Via Ferrata 8, 27100, Pavia, Italy

(Correspondence should be addressed to Massimo Tonacchera, Dipartimento di Endocrinologia, Università degli Studi di Pisa, Via Paradisa 2, 56124, Cisanello, Pisa, Italy; Email: mtonacchera@hotmail.com)

\begin{abstract}
Objective: The TSH receptor (TSHr) expressed on thyroid follicular cells is the autoantigen involved in the pathogenesis of Graves' hyperthyroidism. Whether this receptor is expressed in extrathyroidal tissues, and whether it participates directly in the pathogenesis of thyroid-associated ophthalmopathy (TAO) is unclear.

Design: The aim of the present study was to measure TSHr mRNA in retro-orbital tissues, retro-orbital adipose tissue, extraocular muscle, and skin from patients with TAO and in several tissues from patients not affected by thyroid diseases using RT-PCR and real-time PCR.

Methods: Total RNA was isolated from tissue specimens, reverse transcribed, and amplified using specific primers for the extracellular portion and a part of a $1.3 \mathrm{kbp}$ variant form of the TSHr gene. Determination of TSHr mRNA levels using real-time PCR was also performed by the TaqMan system; to normalize for differences in the amount of total RNA added to the reaction, amplification of $\beta$-actin gene was performed as an endogenous control.

Results: A single-round RT-PCR amplification using specific primers for the extracellular portion of the TSHr gene demonstrated an amplification product of $1.2 \mathrm{kbp}$ in the thyroid, but not in all other tissues. A second-round RT-PCR amplification using the same primers and starting from the previous amplification product demonstrated a band of the size expected for the TSHr gene in all tissue specimens analyzed irrespective of their origin. Similar results were obtained using primers specific for a part of the variant form of $1.3 \mathrm{kbp}$ of the TSHr gene. The amount of TSHr mRNA measured by real-time PCR with the TaqMan probe and expressed as TSHr/ $\beta$-actin ratio was similar in the tissues from TAO patients with respect to the tissues from subjects not affected by thyroid diseases.

Conclusions: We measured TSHr mRNA in tissues from patients with TAO using the very sensitive and quantitative method of real-time PCR. The level of transcription was similar to that measured in extraorbital tissues from patients who were not affected by thyroid diseases. These data suggest an illegitimate TSHr mRNA transcription in all the tissues examined apart from thyroid.
\end{abstract}

European Journal of Endocrinology 147 733-739

\section{Introduction}

Thyroid-associated ophthalmopathy (TAO) is a chronic autoimmune disorder closely associated with Graves' disease $(1,2)$. Rarely TAO may precede the onset of Graves' hyperthyroidism, but in most instances it occurs concurrently with the development of hyperthyroidism (2). Using computed tomography or magnetic resonance scan of the orbit, signs of TAO can be detected in up to $90 \%$ of the patients, but clinical features are evident in only $25-50 \%$ of patients with Graves' disease (2). The different clinical features of TAO, which include proptosis, extraocular muscle dysfunction, periorbital edema and conjunctival injection are due to an inflammatory process of retroorbital tissues leading to an increase in connective tissue and extraocular muscle volume (1). Macroscopic examination of orbital tissues in patients with TAO reveals enlarged extraocular muscle bodies, and increase in orbital connective and fatty tissue volume (1). The close clinical association between immunogenic hyperthyroidism, ophthalmopathy and pretibial dermopathy suggests that the antigen involved in these conditions is shared among the thyroid gland, retro-orbital tissue and pretibial skin. The immune recognition event which causes the $\mathrm{T}$ lymphocytes migrating into the orbit in the first place has not been identified and experimental evidence provides 
arguments both for and against its being the thyrotropin receptor (TSHr) $(1,3)$. Reports concerning the relationship between TSHr antibodies (TRAb) and the severity of ophthalmopathy are also controversial (4). Indeed, many patients with Graves' disease without clinically evident ophthalmopathy have high titers of TRAb in their serum (4). Conversely, some patients with severe TAO do not have detectable TRAb (4). In addition, there is no general agreement regarding the presence of the TSHr or its transcript in eye orbit structures. Some have found transcripts in retro-ocular fibroblasts (5) or inferred their presence in fat (6); others have failed to find a TSHr transcript in retroocular muscle also containing fibroblasts (7). Finally a TSHr variant transcript comprising essentially exons 1-8 was also described in many locations, including ocular muscle, fat, lymphocytes, and fibroblasts (8).

The aim of the present study was to measure, by realtime PCR, TSHr mRNA in retro-orbital adipose tissue, extraocular muscle, and whole retro-orbital tissues from patients with TAO and to compare its expression with that in other extraorbital tissues obtained in patients with no thyroid diseases.

\section{Materials, subjects and methods}

\section{Patients, tissue samples, RNA isolation and reverse transcription}

Whole retro-orbital tissue, retro-orbital adipose tissue, extraocular muscle and skin were obtained during orbital decompression surgery for severe Graves' ophthalmopathy from five patients. Tissue specimens were frozen in liquid nitrogen and stored at $-80^{\circ} \mathrm{C}$ until processed for RNA isolation. One patient underwent orbital decompression for optic neuropathy after 2 days of intravenous administration of high doses of glucocorticoids. The other four patients were submitted to orbital decompression for persistent marked proptosis after a previous course of glucocorticoids and orbital radiotherapy performed 1 year previously. Other tissue specimens (skeletric muscle, abdominal adipose tissue, skin, liver, blood leukocytes, lymph node, brain, pancreas, and gut) were obtained during surgical procedures from patients who did not have thyroid diseases.

Total RNA was isolated from approximately 30$40 \mathrm{mg}$ frozen tissue using the AquaPure RNA Isolation
Kit (Bio-Rad Laboratories, Hercules, CA, USA) according to the manufacturer's instructions. The quality of RNA samples was assessed by electrophoresis through denaturing agarose gels and staining with ethidium bromide to visualize the $18 \mathrm{~S}$ and $28 \mathrm{~S}$ RNA bands under UV illumination. The extraction yield was quantified spectrophotometrically at $260 \mathrm{~nm}$.

One microgram of total RNA for each sample was reverse transcribed for $1 \mathrm{~h}$ at $50^{\circ} \mathrm{C}$ in a $20 \mu \mathrm{l}$ reaction volume using 200 units Superscript II Rnase $\mathrm{H}^{-}$ reverse transcriptase (Life Technologies Inc., Gaithersburg, MD, USA) in the presence of $1.5 \mu \mathrm{M}$ random examers (Pharmacia Biotech, Uppsala, Sweden), $0.01 \mathrm{M}$ dithiothreitol and $1 \mathrm{mM}$ dNTP mix.

\section{PCR amplification}

Five hundred nanograms of reverse-transcribed cDNA were amplified using specific primers for the entire extracellular part of the TSHr and a $714 \mathrm{bp}$ part of a $1.3 \mathrm{kbp}$ variant form of the TSHr gene (8). The sequence and the annealing temperature of the primers, and the length of the amplified fragments are shown in Table 1. The same amount of cDNA was also used to amplify a fragment of the $\beta$-actin gene. To rule out genomic DNA contamination, PCR primer pairs crossed exons and PCR of not reverse-transcribed RNA yielded no specific bands. PCR amplifications were carried out in $100 \mu \mathrm{l}$ reaction mixture containing $20 \mathrm{pM}$ specific primers and $1.5 \mathrm{mM} \mathrm{MgCl}_{2}$ in the presence of $1 \mathrm{U}$ Taq DNA Polymerase (Life Technologies Inc.). Second-round PCR amplifications were carried out following the protocol above, but starting from $10 \mu \mathrm{l}$ of the previous amplification product as a template. Following the amplification, $10 \mu \mathrm{l}$ of the reaction mix was separated on a $1 \%$ agarose gel and the resulting bands were visualized in UV light on a transilluminator, following ethidium bromide staining. Molecular weights were compared with a $100 \mathrm{bp}$ ladder.

\section{Determination of TSHr mRNA levels using real-time PCR}

Oligonucleotide primers and TaqMan probes were designed to be intron spanning, using the computer program Primer Express (Applied Biosystems, Foster City, CA, USA). To avoid amplification of contaminating genomic DNA, one of the two primers or the probe was

Table 1 PCR primers used to amplify the extracellular portion (ECD) of the TSHr gene and a part of the $1.3 \mathrm{kbp}$ of the variant form of the TSHr gene.

\begin{tabular}{lll}
\hline ECD forward & GGCGGAATGGGGTGTTCGTC & Annealing temperature $=55^{\circ} \mathrm{C}$ \\
ECD reverse & GAACTTGTAGCCCATTATGT & Fragment length $=1191 \mathrm{bp}$ \\
ECD forward & GGCGGAATGGGGTGTTCGTC & Annealing temperature $=55^{\circ} \mathrm{C}$ \\
Variant reverse & GGACTTTCTTCCAAGAGG & Fragment length $=714 \mathrm{bp}$ \\
\hline
\end{tabular}


Table 2 Oligonucleotide sequence of the primers and the TaqMan hybridization probe for real-time PCR to quantitate TSHr cDNA.

Primer forward CCCAGCTTACCGCCCAGT

Primer reverse TAGAAAATGCATGACTTGGAATAGTTC Probe CGCAGACTGTGAAGCTTATTGAGACTCACCTG

placed at the junction between two exons, or in a different exon. The nucleotide sequence of the primers and probes are shown in Table 2 and they were purchased from Applied Biosystems.

Quantitative PCR reaction was carried out in 96-well optical reaction plates using a cDNA equivalent of $50 \mathrm{ng}$ total RNA for each sample in a volume of $50 \mu \mathrm{l}$ using the TaqMan Universal PCR Master Mix (Applied Biosystems) according to the manufacturer's instructions. PCR was developed on the ABI Prism 7700 Sequence Detector (Applied Biosystems). The thermal cycling conditions comprised an initial denaturation step at $95^{\circ} \mathrm{C}$ for $10 \mathrm{~min}$ and 40 cycles of two-step PCR, including $15 \mathrm{~s}$ of denaturation at $95^{\circ} \mathrm{C}$ and $1 \mathrm{~min}$ of annealing-elongation at $60^{\circ} \mathrm{C}$, using the standard protocol of the manufacturer. Each sample was assayed in triplicate and the intra-assay coefficient of variation was less than $1 \%$. Experiments were repeated three times. The monitoring of negative controls for each target showed an absence of carryover.

To minimize the errors arising from the variation in the amount of starting RNA among samples, amplification of $\beta$-actin mRNA was performed as an internal reference against which other RNA values can be normalized. The primers and the probe for the $\beta$-actin RNA were purchased from Applied Biosystems.

Normalized results were expressed as the ratio of pg RNA of the TSHr gene to pg RNA of the $\beta$-actin gene (means \pm S.E. of three experiments).

\section{Statistical analysis}

The expression of the TSHr mRNA detected in the different pathological tissues from patients with TAO was compared with respect to its expression in extrathyroidal tissues from subjects not affected by thyroid diseases using the Student's $t$-test; to test the significance, the risk level $(P)$ was set at 0.05 .

\section{Results}

\section{RT-PCR amplification}

RT-PCR for $\beta$-actin was performed in all the tissues examined to verify both the integrity of the RNA preparation and the success of the RT step. In all samples tested, a single-step RT-PCR amplification demonstrated the specific band (data not shown).
A single round of RT-PCR amplification using specific primers for the extracellular part of the TSHr gene demonstrated the amplification product of about $1.2 \mathrm{kbp}$ only in the thyroid (positive control), but not in all other tissue specimens examined (data not shown). A second-round RT-PCR amplification using the same primers but starting from $10 \mu \mathrm{l}$ of the previous amplification product demonstrated a band of the expected size in all samples analyzed (Fig. 1A). Using this technique, additional amplification bands of different sizes could also be visualized. Similar results were obtained using the primers specific for a part of $714 \mathrm{bp}$ of a $1.3 \mathrm{kbp}$ variant form of the TSHr gene (Fig. 1B).

\section{Real-time PCR}

To validate the real-time PCR method, a standard curve for the TSHr gene was constructed from cDNA obtained from normal thyroid tissue (Fig. 2). Total RNA was isolated from normal thyroid tissue and, after its quantification by spectophotometry, $5 \mu \mathrm{g}$ was reverse transcribed as described above. The cDNA was serially diluted in nuclease-free water to produce a standard curve that ranged from 1.0 to $10000 \mathrm{pg}$. Figure 2 shows the real-time PCR standard curve for the TSHr gene. The efficiency of the standard curve, as determined by its slope and correlation coefficient, allowed us to quantify the gene expression profile in each specimen by using the comparative threshold cycle method according to the manufacturer's instructions. A standard curve using the same cDNAs was also constructed for the $\beta$-actin gene (data not shown). For each experimental sample, the amount of the targets and the endogenous reference was determined from the standard curve. The target amount was then divided by the endogenous reference amount to obtain a normalized target value.

Absolute quantification of mRNA for the TSHr and the $\beta$-actin genes showed similar expression levels in the samples obtained from patients with TAO with respect to the tissues obtained from subjects not affected by thyroid diseases (Fig. 3A and B). After normalization for the expression of the endogenous gene, the $\mathrm{TSHr} / \beta$-actin ratio was similar in tissues from patients with TAO with respect to the tissues obtained from subjects not affected by thyroid diseases (Fig. 3C). No statistically significant difference was observed for the expression of the TSHr mRNA in tissues from TAO patients with respect to tissues from patients not affected by thyroid diseases.

The ratio of TSHr/ $\beta$-actin measured in normal thyroid tissue (obtained from the contralateral lobe in the course of thyroidectomy for thyroid carcinoma) was 0.38; the other samples analyzed ranged from 0.0004 to 0.09 (Fig. 4). 
(A)

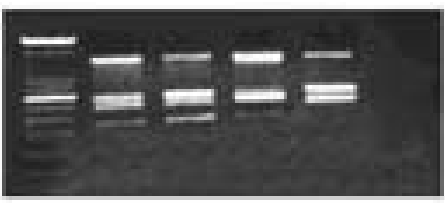

\section{1 bp}

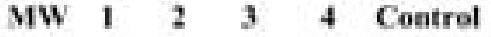
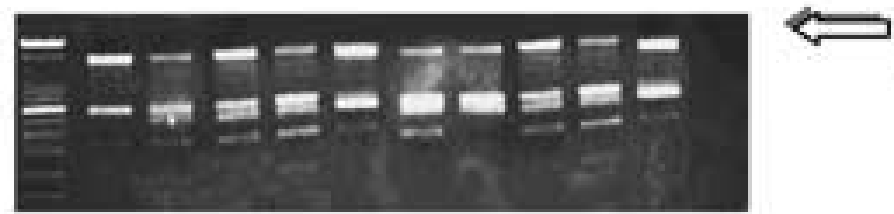

1191 bp

(B)

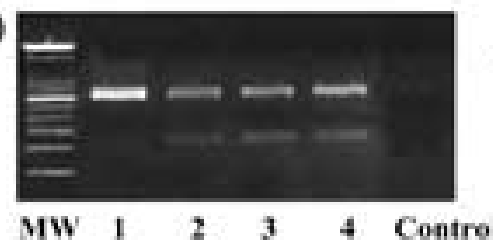

\section{4 bp}

$\begin{array}{llllll}\text { MIW } & 1 & 2 & 3 & 4 & \text { Control }\end{array}$
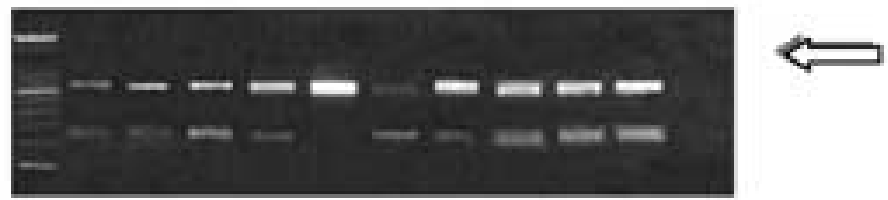

714 bp

$\begin{array}{llllllllllll}\text { MW } & 5 & 6 & 7 & 8 & 9 & 10 & 11 & 12 & 13 & 14 & \text { Control }\end{array}$

Figure 1 Second-round RT-PCR amplification of the extracellular part of the TSHr cDNA yielding a $1.2 \mathrm{kbp}$ fragment $(\mathrm{A})$ and of a part (714 bp) of a $1.3 \mathrm{kbp} \mathrm{TSHr}$ variant (B) of cDNAs from different tissues: 1 , whole retro-orbital tissue; 2 , adipose tissue; 3 , extraocular muscle; 4 , skin (all these tissues from one patient with TAO); 5 , abdominal adipose tissue; 6 , skeletric muscle; 7 , skin; 8 , liver; 9, lymph node; 10, pancreas; 11, gut; 12, blood leukocyte; 13 , brain; 14, thyroid. The PCR products were separated on a $1.0 \%$ agarose gel with a 100 bp ladder as size marker.

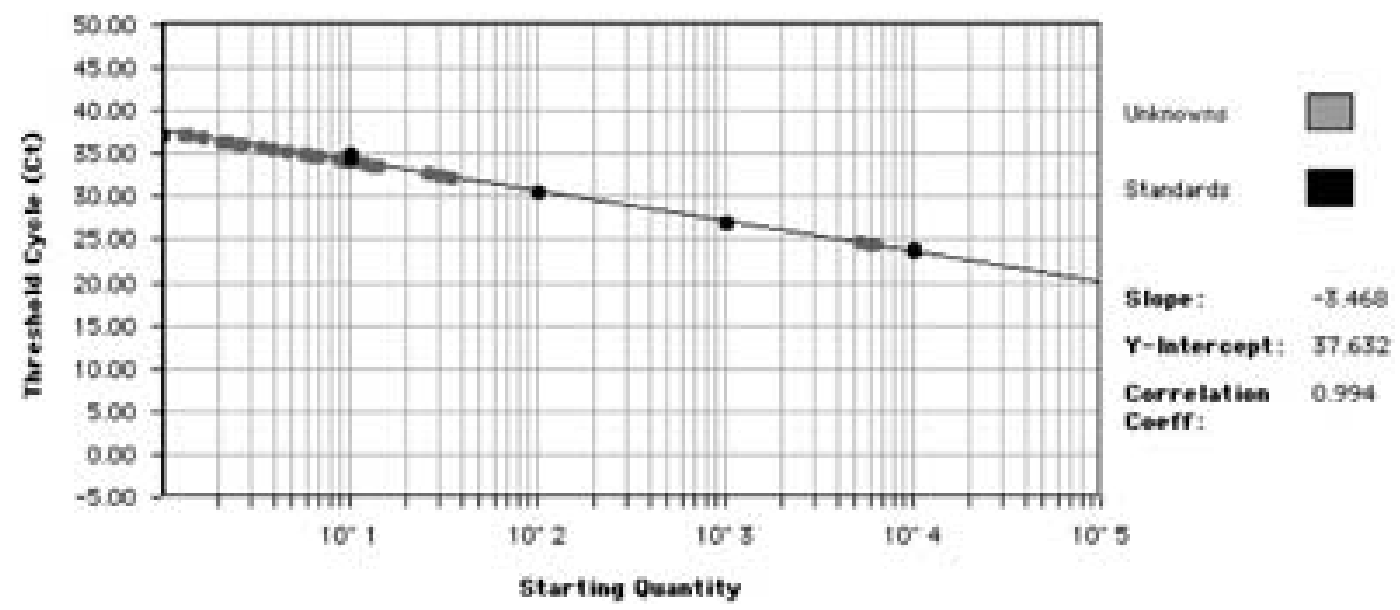

Figure 2 Calibration curve for TSHr mRNA using the TaqMan PCR analysis. A standard curve plotting the log of the input amount $(1,10,100,1000$, and $10000 \mathrm{pg}$ of normal starting RNA) vs the threshold cycle (Ct) was determined as described in the Results. The threshold cycle represents the PCR cycle at which an increase in reporter fluorescence above a baseline signal was first detected. 

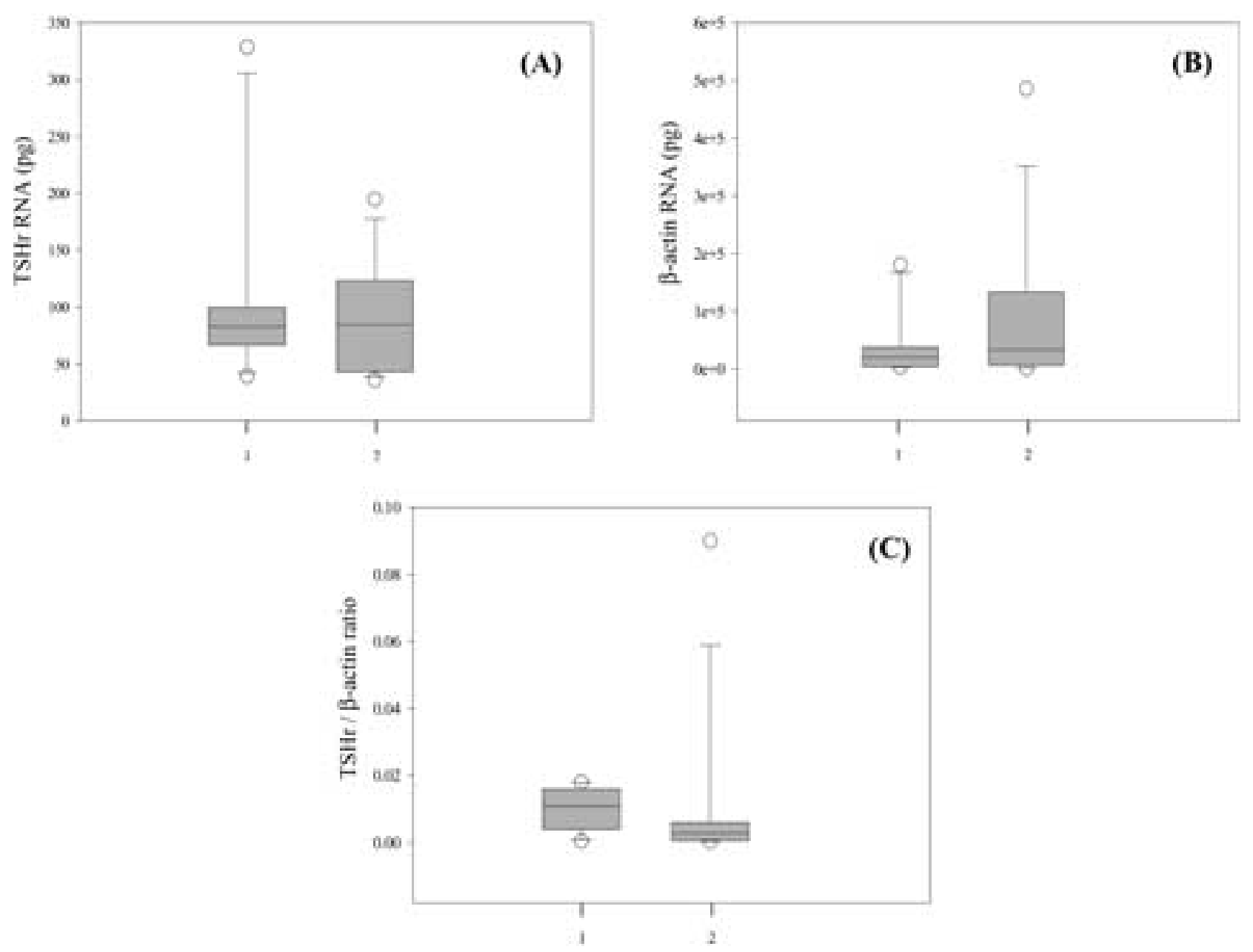

Figure 3 Box plot showing the distribution of mRNA values for TSHr (A), $\beta$-actin (B) and TSHr/ $\beta$-actin ratio $(C)$ in tissues from TAO patients (1) and tissues from patients not affected by thyroid diseases (2). The upper and lower limits of the boxes represent the 75 th and 25 th percentile respectively; the lines inside the boxes indicate the median. The upper and lower whiskers indicate 90 th and 10 th percentiles respectively. Outliers are illustrated as open circles.

\section{Discussion}

The TSHr expressed on thyroid follicular cells is the autoantigen involved in the pathogenesis of Graves' hyperthyroidism $(1-3)$. Whether this receptor is expressed in extrathyroidal tissues, and whether it participates directly in the pathogenesis of TAO is unclear $(1-3)$.

Using conventional single-round RT-PCR amplification we were able to amplify a $1.2 \mathrm{kbp}$ band specific for the extracellular portion of the TSHr only in the thyroid tissue. Using further amplification steps and starting from the previous PCR product as a template the expected band was also found in the extrathyroidal tissues (both orbital and non-orbital) from TAO patients and controls. Similarly, after many cycles of PCR amplification, we obtained the band corresponding to a part of the previously described variant form of the TSHr (8). These results suggest the presence of a very low level of
TSHr mRNA not only in the retro-orbital adipose tissue, extraocular muscle, the whole retro-orbital tissue and the skin from patients with TAO, but also in the other extraorbital tissues. In order to obtain a more accurate measure of the mRNA of the TSHr gene, a real-time PCR was performed. Real-time PCR is a novel technique that measures PCR product accumulation during the exponential phases of PCR reaction (9). With this method, it is possible to monitor PCR product accumulation cycle by cycle, thus providing accurate measurement of target concentration over a wide range of starting materials (9).

To validate the real-time PCR method, a standard curve for the TSHr gene was constructed from cDNA obtained from normal thyroid tissue. To quantify the gene expression profile in each specimen, we used the comparative threshold cycle method. The real-time quantitative PCR method showed the transcript of the TSHr gene in all the samples analyzed. Starting from 


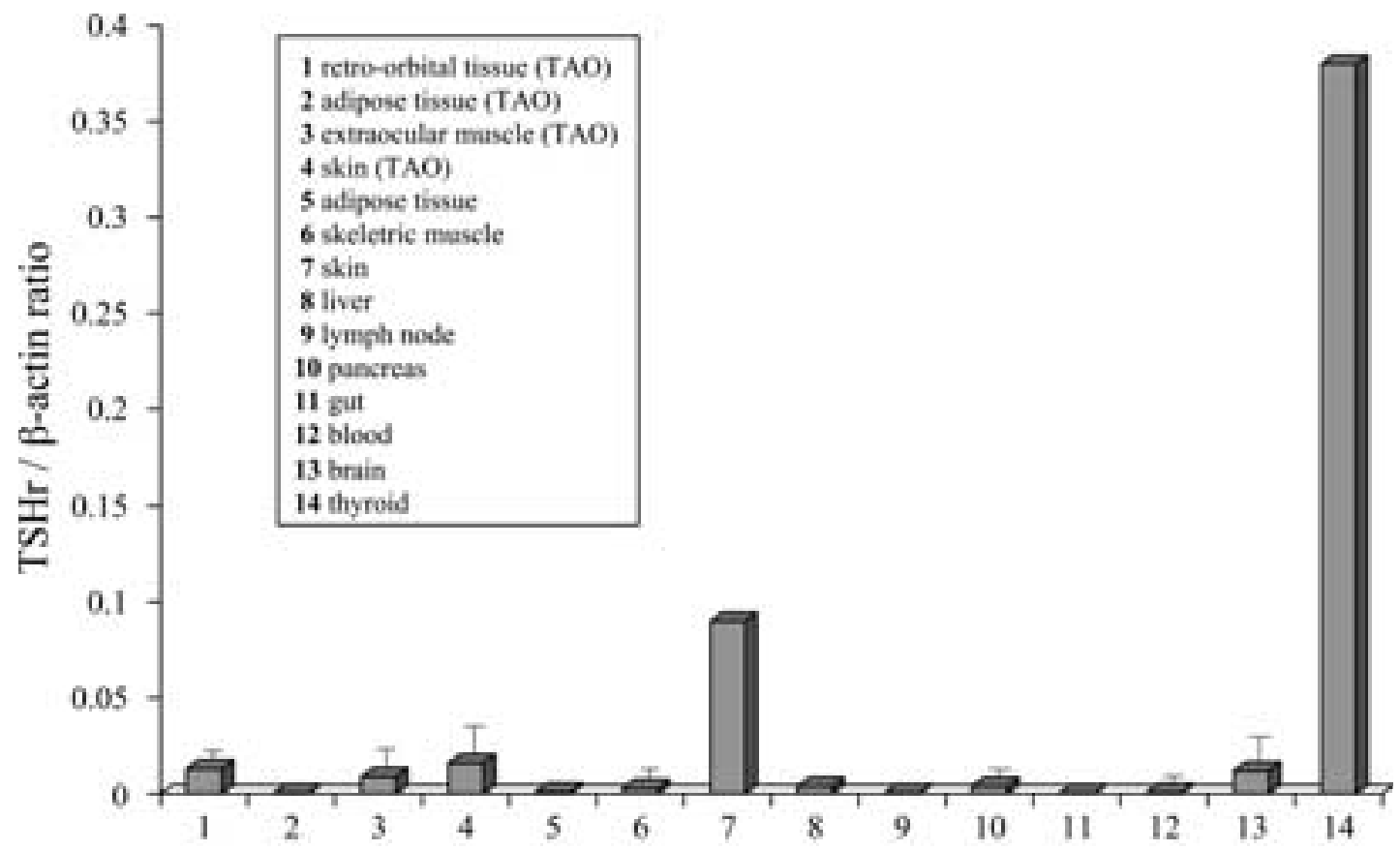

Figure $4 \mathrm{TSHr}$ mRNA levels measured by real-time PCR in the retro-orbital tissues of one of the five patients with TAO and in the various tissues that were examined expressed as TSHr/ $\beta$-actin ratio as described in Materials, subjects and methods. Each bar represents the mean \pm S.E. of three independent experiments; where error bars are not visible, they are so small that they fall within the symbols.

$1 \mu \mathrm{g}$ RNA for each tissue sample, we measured a huge difference between the thyroid tissue and the other tissues that we analyzed. Results for the TSHr mRNA expression were normalized with respect to the $\beta$-actin mRNA. After normalization, no statistically significant difference was observed for the expression of the TSHr mRNA in tissues from TAO patients with respect to tissues from patients not affected by thyroid diseases.

Several groups of investigators have sought evidence for TSHr expression in various orbital tissues $(5-8$, 10-17). Attempts to identify TSHr mRNA in these tissues using Northern blotting have proved largely unsuccessful, although a recent study (11) showed positive bands in a single specimen after a very long exposure period. In contrast, several authors have detected TSHr mRNA or a variant transcript in human orbital tissues and cell cultures of orbital fibroblast using RT-PCR (5-8). RNA transcripts that can be detected only by PCR- based amplification of cDNA may have little physiological relevance.

Recently Bahn et al. (12), by using liquid hybridization analysis, showed the presence of mRNA of the TSHr in the adipose/connective tissue of the diseased orbit in patients with TAO. In addition, TSHr was demonstrable in early passage TAO preadipocite orbital fibroblast cultures that contained a subpopulation of adipocytes. Subsequent passage of these cells resulted in the loss of TSHr expression. The authors concluded that the expression of this receptor in TAO may be induced in vivo by a humoral factor not present in the cell culture environment $(12,13)$. Recently, gene expression profiles in extraocular muscles in adult mice by high-density oligonucleotide arrays were analyzed (18). The use of significance analysis of microarray methodology identified up to 400 genes as having an extraocular-specific expression pattern and the TSHr was not shown (18).

To our knowledge this is the first time that TSHr mRNA has been measured by a sensitive and quantitative method such as real-time PCR in retro-orbital tissues from patients with TAO.

The results obtained in this paper show that the TSHr transcript is present in retro-orbital tissue (both the adipose and the whole retro-orbital), extraocular muscle and the skin from patients with TAO. A comparable amount of TSHr mRNA transcript was also measured in different tissues from patients without thyroid diseases. These data support the view that TSHr mRNA expression in extrathyroid tissues could represent an illegitimate transcription (19). Illegitimate transcription is produced as a result of leaky transcription, i.e. transcription that presumably occurs incidentally rather than intentionally (19). This conclusion can be drawn for the very low level of TSHr mRNA transcript measured both in the tissues of patients with TAO and in the tissues of patients without thyroid diseases which were examined. 
Whether this low level of expression of TSHr in extrathyroid tissues could have relevance in the pathogenesis of TAO and pretibial mixedema remains to be elucidated.

It has been proposed that even tiny quantities of appropriately processed and presented antigen may be sufficient to stimulate recognition by the immune system (20). According to this hypothesis, Graves' disease might represent a generalized connective tissue process that becomes clinically evident in specific areas of the body where either a confined space (the orbit) or continuous trauma (pretibial area) favors the development of a full-blown inflammatory process. We might also speculate that, in particular situations, local factors could determine a TSHr expression that could mediate some of the clinical and pathological manifestations of TAO (20).

In conclusion, we measured TSHr mRNA from pathological tissues from patients with TAO by using the very sensitive and quantitative method of realtime PCR. The level of transcription was similar to that measured in other tissues from patients who were not affected by thyroid diseases. These data support the notion of an illegitimate TSHr mRNA transcription in all the tissues examined.

\section{Acknowledgements}

This work was supported by the following grants: Ministero dell'Università e della Ricerca Scientifica (MURST), Programma di Ricerca: Meccanismi molecolari nella patogenesi del nodulo tiroideo; MURST, Programma di Ricerca: Strategie per la valutazione degli effetti disruptivi dei contaminanti ambientali sul sistema endocrino degli animali e dell'uomo; MURST, Programma di Ricerca: Oftalmopatia basedowiana: analisi dei fattori patogenetici, nuovi approcci diagnostici e terapeutici, efficacia e potenziali rischi a lungo termine dei trattamenti tradizionali.

\section{References}

1 Bahn RS \& Heufelder AE. Pathogenesis of Graves' ophthalmopathy. New England Journal of Medicine 199311 1468-1474.

2 Marcocci C, Bartalena L, Bogazzi F, Panicucci M \& Pinchera A. Studies on the occurence of ophthalmopathy in Graves' disease. Acta Endocrinologica $1989120473-478$.

3 Paschke R, Vassart G \& Ludgate M. Current evidence for and against the TSH receptor being the common antigen in Graves' disease and thyroid associated ophthalmopathy. Clinical Endocrinology $199542565-569$.

4 Rapoport B, Chazenbalk GD, Juame JC \& McLachlan SM. The thyrotropin receptor: interaction with TSH and autoantibodies. Endocrine Reviews $199819673-716$.

5 Heufelder AE, Dutton CM, Sarkar G, Donovan KA \& Bahn RS. Detection of TSH receptor RNA in cultured fibroblasts from patients with Graves' ophthalmopathy and pretibial dermopathy. Thyroid 19933 297-300.

6 Feliciello A, Porcellini A, Ciullo I, Bonavolonta G, Avvedimento EV \& Fenzi G. Expression of thyrotropin receptor mRNA in healthy and Graves' disease retro-orbital tissue. Lancet $1993 \mathbf{3 4 2}$ 337-338.

7 Paschke R, Elisei R, Vassart G \& Ludgate M. Lack of evidence supporting the presence of mRNA for the thyrotropin receptor in extra-ocular muscle. Journal of Endocrinological Investigation $199316329-332$

8 Paschke R, Metcalfe A, Alcalde L, Vassart G, Weetman A \& Ludgate M. Presence of nonfunctional thyrotropin receptor variant transcripts in retroocular and other tissues. Journal of Clinical Endocrinology and Metabolism 199479 1234-1238.

9 Bustin SA. Absolute quantification of mRNA using real-time reverse transcription polymerase chain reaction assays. Journal of Molecular Endocrinology 200025 169-193.

10 Perros P \& Kendall-Taylor P. Demonstration of thyrotropin binding sites in orbital connective tissue: possible role in the pathogenesis of thyroid associated ophthalmopathy. Journal of Endocrinological Investigation 199417 163-170.

11 Crisp MS, Lane C, Halliwell M, Wyndford-Thomas D \& Ludgate M. Thyrotropin receptor transcripts in human adipose tissue. Journal of Clinical Endocrinology and Metabolism 199782 2003-2005.

12 Bahn RS, Dutton CM, Natt N, Joba W, Spitzweg C \& Heufelder AE. Thyrotropin receptor expression in Graves' orbital adipose/connective tissues: potential autoantigen in Graves' ophthalmopathy. Journal of Clinical Endocrinology and Metabolism $1998 \mathbf{8 3}$ 998-1002.

13 Jyonouchi SC, Valyasevi RW, Harteneck DA, Dutton CM \& Bahn RS. Interleukin-6 stimulates thyrotropin receptor expression in human orbital preadipocyte fibroblasts from patients with Graves' ophthalmopathy. Thyroid $2001 \quad 11$ 929-934.

14 Umit Bagriaçik E \& Klein JR. The thytropin (thyroid-stimulating hormone) receptor is expressed on murine dendritic cells and on a subset of CD45RB high lymph node T cells: functional role for thyroid-stimulating hormone during immune activation. Journal of Immunology $2000 \mathbf{1 6 4} 6158-6165$.

15 Smith TJ, Koumas L, Gagnon A, Bell A, Sempowski GD, Phipps RP et al. Orbital fibroblast heterogeneity may determine the clinical presentation of thyroid-associated ophthalmopathy. Journal of Clinical Endocrinology and Metabolism 200287 385-392.

16 Valyasevi RW, Erickson DZ, Harteneck DA, Dutton CM, Heufelder AE et al. Differentiation of human orbital preadipocyte fibroblasts induces expression of functional thyrotropin receptor. Journal of Clinical Endocrinology and Metabolism 1999 $842257-2562$.

17 Busuttil BE \& Frauman AG. Extrathyroidal manifestations of Graves' disease: thyrotropin receptor is expressed in extraocular, but not cardiac, muscle tissues. Journal of Clinical Endocrinology and Metabolism 200186 2315-2319.

18 Porter JD, Khanna S, Kaminski HJ, Rao JS, Merriam AP, Richmonds CR et al. Extraocular muscle is defined by a fundamentally distinct gene expression profile. PNAS $2001 \quad 98$ 12062-12067.

19 Chelly J, Concordet JP, Kaplan JC \& Kahn A. Illegitimate transcription: transcription of any gene in any cell type. PNAS $1989 \mathbf{8 6}$ 2617-2621.

20 Tomer Y \& Davies TF. The genetic susceptibility to Graves' disease. Baillieres Clinical Endocrinology and Metabolism $1997 \quad 11$ $431-450$.

Received 16 May 2002

Accepted 16 September 2002 